\title{
SYNTHESIS AND CHARACTERIZATION OF PH-SENSITIVE N-SUCCINYL CHITOSAN HYDROGEL AND ITS PROPERTIES FOR BIOMEDICAL APPLICATIONS
}

\author{
SHAHID BASHIR ${ }^{a, b}$, YIN YIN TEO $^{a}$, S. RAMESH ${ }^{b^{*}}$, K. RAMESH $^{b}$, MUHAMMAD RIZWAN $^{a}$ AND \\ MUHAMMAD RIZWAN ${ }^{a, c}$ \\ ${ }^{a}$ Department of Chemistry, Faculty of Science, University of Malaya, Kuala Lumpur 50603, Malaysia. \\ ${ }^{b}$ Centre of Ionics University of Malaya, Department of Physics, Faculty of Science, University of Malaya, Kuala Lumpur 50603, \\ Malaysia. \\ ${ }^{c}$ Department of Chemistry, The University of Lahore, Lahore, Pakistan.
}

\begin{abstract}
Natural polymers have been widely used in different fields specifically in pharmaceutical industry due to their biocompatibility, biodegradability, and less toxicity properties. Among these polymers, chitosan is a potentially pursued biomaterial. However, limited solubility of chitosan in water creates significant barrier in realizing its applications as a biomaterial. Modification of chitosan helps to overcome this barrier with improved solubility in water. In this study, $\mathrm{N}$-succinyl chitosan was synthesized from the chemical reaction of chitosan and succinic anhydride via Schiff base mechanism by using glutaraldehyde as a crosslinking agent. N-succinyl chitosan is water soluble, biocompatible, biodegradable, and less toxic. The physico-chemical properties of NSC and prepared hydrogel were characterized by using Fourier transform infrared (FTIR) spectroscopy, Nuclear Magnetic Resonance Spectroscopy (NMR), X-ray diffraction (XRD) analysis, and differential scanning calorimetry (DSC). The surface morphology was investigated through Field emission scanning electron microscopy (FESEM). All these results confirmed the successful synthesis of $\mathrm{N}$-succinyl chitosan and its hydrogel. Moreover, the mechanical properties of the hydrogel were evaluated using rheometer and found that hydrogel has significant potential to be used in the biomedical field. In addition, the swelling properties of the hydrogel were also studied in buffer solutions of $\mathrm{pH}$ 1.2 and 7.4. These results endorsed its applications as a drug delivery carrier.
\end{abstract}

Keywords: Hydrogel, mechanical and swelling properties, N-Succinyl chitosan.

\section{INTRODUCTION}

Hydrogels are three-dimensional hydrophilic polymeric networks. Hydrogels may perhaps chemically stable or may degrade and ultimately disintegrate and dissolve. They are called physical gels when their networks are detained together by secondary forces including hydrogen bonding, ionic or hydrophobic forces and molecular entanglements [1]. Formation of the physical gels through clustering of molecules can cause inhomogeneities [2]. Free chain loops signify short-lived network imperfection in physical gels. Hydrogels are called chemical gels when polymer networks are crosslinked via covalent bonds. These gels are also known as permanent gels [3]. The chemical gels can be synthesized via crosslinking of hydrophilic polymers or hydrophobic polymers by first convert into hydrophilic and then crosslinked to form network. Various macromolecular networks are probable for formation of physical and chemical gels such as molecules entanglement or crosslinking network structures between homopolymers, linear copolymers, block or grafted copolymers, polyionpolyion, polyion-multivalent ion or hydrogen bonded complexes [4]. Hydrogels possess different physical appearances like solid, pressed powder pills or capsules, microparticles, membranes or sheets. Furthermore, hydrogels can imbibe and retain large quantity of water and swells up to thousands of times their initial dry weight. Due to high water contents, hydrogels are soft, flexible in fabrication with desirable characteristics. Hydrogels also respond to external stimuli such as $\mathrm{pH}$, temperature, salt, light, electromagnetic field, and biomolecules [5].

A diverse range of polymer compositions have been used to fabricate hydrogels [6]. The compositions can be divided into natural, synthetic or combination of two classes. Hydrogels comprised of natural polymers have vast range of applications in the routine applications and in the biomedical field due to biocompatibility, biodegradability, and non-toxic nature. Among the natural polymers, chitosan is one such semi-synthetic hetero-polymer of D-glucosamine and $\mathrm{N}$-acetyl D-glucosamine units. This is cationic in nature and obtained from deacetylation of chitin which is the most important component of the exoskeleton of crustacean, such as shrimps [7]. Currently, chitosan attracted striking attention in medical and pharmaceutical applications due to its interesting intrinsic properties [8]. The main parameter controlling the properties of chitosan is its molecular weight. Chitosan is water insoluble due to its high molecular weight, but it is soluble in aqueous solution of acetic acid by forming strong intermolecular hydrogen bonding [9]. Hydrogels fabricated from chitosan often require repeated washing to neutralize and eradicate excess acid which restricts its applications in the biomedical field. In order to overcome the limitations, interest in modification of chitosan has been growing because water soluble derivatives of chitosan are attractive to biomedical applications. One such water soluble derivative is $\mathrm{N}$-succinyl chitosan, at present in enormous demand.
This derivative has already been exploiting as cosmetic ingredient (Moistfine liquid(B) [10]. This chitosan derivative is highly $\mathrm{pH}$-sensitive and has prolonged circulation time in the human body [11]. Furthermore, the negatively charge of carboxylate group in $\mathrm{N}$-succinyl chitosan prompotes the properties of mucoadhesive. It exhibits anticoagulating and antiaggregating properties and does not change the parameters of system hemodynamics and shows very low cytotoxicity to normal tissues [12]. It promotes the adhesion, proliferation, and differentiation of cells and evokes a minimum foreign body reaction upon implantation. It is osteoconductive and has the ability to enhance bone formation in vitro and in vivo. $\mathrm{N}$-succinyl chitosan is a very useful material in the biomedical field [13]

In the present work, $\mathrm{N}$-succinyl chitosan was synthesized from chitosan. Then, NSC hydrogel was prepared via Schiff-base mechanism using glutaraldehyde (GA) as a crosslinking agent. The physico-chemical properties were characterized using FTIR, NMR, XRD, and DSC techniques. The surface morphology of synthesized hydrogel was observed through field emission scanning electron microscope (FESEM). The mechanical properties were investigated using rheometer and swelling properties were examined in buffer solutions of $\mathrm{pH} 1.2$ and $\mathrm{pH} 7.4$.

\section{MATERIALS AND METHODS}

\subsection{Materials}

Chitosan (medium molecular weight $\left(\mathrm{M}_{\mathrm{w}}\right.$ 190-310 kDa), degree of deacetylation $75-85 \%$, viscosity $=200-800 \mathrm{cP}$ ), and succinic anhydride were obtained from Sigma Aldrich (Malaysia). Glutaraldehyde (25\% solution) obtained from Unilab, sodium hydroxide, acetic acid, acetone, and methanol were purchased from Friendemann Schmidt (Malaysia), ethanol (John Koillin chemicals), Oxalic acid, sodium chloride, potassium dihydrogen phosphate, and potassium chloride were purchased from Univar. All these reagents were used as received.

\subsection{Methods}

\subsubsection{Synthesis of $\mathrm{N}$-succinyl chitosan}

$\mathrm{N}$-succinyl chitosan was prepared according to the reported method with slight modification [14]. $0.6 \mathrm{~g}$ of chitosan was dissolved in $100 \mathrm{ml}$ of $3 \%$ acetic acid and stirred for $45 \mathrm{~min}$ at $50{ }^{\circ} \mathrm{C}$. Then, $50 \mathrm{ml}$ methanol was added to dilute the solution followed by dropwise addition of already dissolved $2.5 \mathrm{~g}$ succinic anhydride in $40 \mathrm{ml}$ acetone. The mixture was stirred at $1400 \mathrm{rpm}$ at $50{ }^{\circ} \mathrm{C}$ for 48 hours. After 48 hours, reaction mixture was diluted with excess $1 \mathrm{M} \mathrm{NaOH}$ solution until clear solution was obtained. 
The clear solution was kept under stirring for 24 hours at $50{ }^{\circ} \mathrm{C}$. Then, ethanol was added to precipitate the product followed by filtration to separate the precipitates. The precipitates were purified by redispersing in ethanol for 24 hours and washed with ethanol and acetone several times to remove the excess of reactants. Pure product was dried in vacuum oven for 12 hours at $50{ }^{\circ} \mathrm{C}$.

\subsubsection{Synthesis of $\mathrm{N}$-succinyl chitosan hydrogel}

$\mathrm{N}$-succinyl chitosan hydrogel was prepared using glutaraldehyde as a crosslinking agent. In order to prepare hydrogel, $3 \mathrm{ml}$ NSC (3\%) was pipetted into a vial followed by addition of $0.3 \mathrm{ml} \mathrm{GA}(1 \%)$. The mixture was mixed thoroughly and kept at $50{ }^{\circ} \mathrm{C}$. The hydrogel was formed immediately. The synthesized hydrogel was taken out from the vial, washed with deionized water, and dried in vacuum oven for 24 hours for further studies.

\subsection{Characterization of the hydrogel}

FTIR studies of chitosan, NSC, and NSC hydrogel were carried out by using FTIR (Perkin Elmer spectrum 400) spectrometer from 4000 to $500 \mathrm{~cm}^{-1}$ at $25^{\circ} \mathrm{C}$.

${ }^{1} \mathrm{H}$ and ${ }^{13} \mathrm{C}$ NMR spectra of NSC were recorded by Bruker $600 \mathrm{MHz}$ spectrometer at room temperature and samples were dissolved in $\mathrm{D}_{2} \mathrm{O}$. The position of $\mathrm{D}_{2} \mathrm{O}$ peak was taken as an internal reference.

X-ray diffraction analysis of chitosan, NSC and NSC hydrogel was performed by X-ray diffractometer (Panalytical Empyrean diffractometer). Samples were exposed to $\mathrm{Cu} \mathrm{K} \alpha$ radiation and scan rate was set at $1^{\circ} \min ^{-1}$ at $2 \theta$.

DSC thermograms were recorded using a differential scanning calorimetry (Q20) over temperature range of $25-400{ }^{\circ} \mathrm{C}$ at the scanning rate of $10{ }^{\circ} \mathrm{C} / \mathrm{min}$. The samples $(5-10 \mathrm{mg})$ were put in aluminum pans and scanned under nitrogen atmosphere.

The surface morphology of the hydrogels was examined using field emission scanning electron microscope (FESEM), Quanta FEG 450. The hydrogel was swelled, cut into small pieces to expose inner surface, then freeze dried using freeze dryer to remove water without disturbing the morphology. After this, the hydrogel was coated with gold in order to prevent the charging effects at an accelerating voltage of $5 \mathrm{kV}$.

\subsection{Rheology study}

The rheological characteristics of the hydrogel were performed using oscillatory MCR 301 rheometer (Paar-Physica) at $25^{\circ} \mathrm{C}$ with $40 \mathrm{~mm}$ in diameter parallel plate geometry. The gap between the upper plate and sample dish was set at $0.5 \mathrm{~mm}$. The force was applied slowly by lowering the upper plate and stopped at $0.1 \mathrm{~N}$. The elasticity modulus G' (storage modulus) and viscosity modulus G" (loss modulus) measurements were performed as a function of frequency at strain amplitude of $0.1 \%$ in order to maintain a linear viscoelastic regime.

\subsection{Swelling ratio study}

The swelling ratio of synthesized hydrogel was observed in buffer solutions of $\mathrm{pH} 1.2$ and $\mathrm{pH} 7.4$ at $37^{\circ} \mathrm{C}$. For this purpose, constant quantity of dried hydrogel was immersed in fixed volume of buffer solutions. At predetermined time intervals, hydrogels were taken out, blotted off and weighted. The weighted hydrogels were soaked again in buffer solutions. This process continued until constant weight was observed.

$$
\mathrm{SR}=\frac{\mathrm{W}_{\mathrm{t}}-\mathrm{W}_{0}}{\mathrm{~W}_{0}}
$$

Where, $\mathrm{W}_{\mathrm{o}}$ is the initial weight of hydrogel and $\mathrm{W}_{\mathrm{t}}$ is the weight of the hydrogel at time $\mathrm{t}$.

\section{RESULTS AND DISCUSSION}

\subsection{Synthesis of NSC and hydrogel}

The fluffy NSC was synthesized successfully and succinylation reaction of chitosan was verified through different characterization techniques. This succinylation reaction occurred through substitution of $\mathrm{H}$ from amino group, forming an amidic bond with opening of the anhydride ring.
Furthermore, hydrogel was formed via Schiff base mechanism which occurred between remaining amino groups of NSC and aldehyde groups of GA. The reaction mechanism of NSC synthesis from chitosan and hydrogel formation is shown in Figure 1.

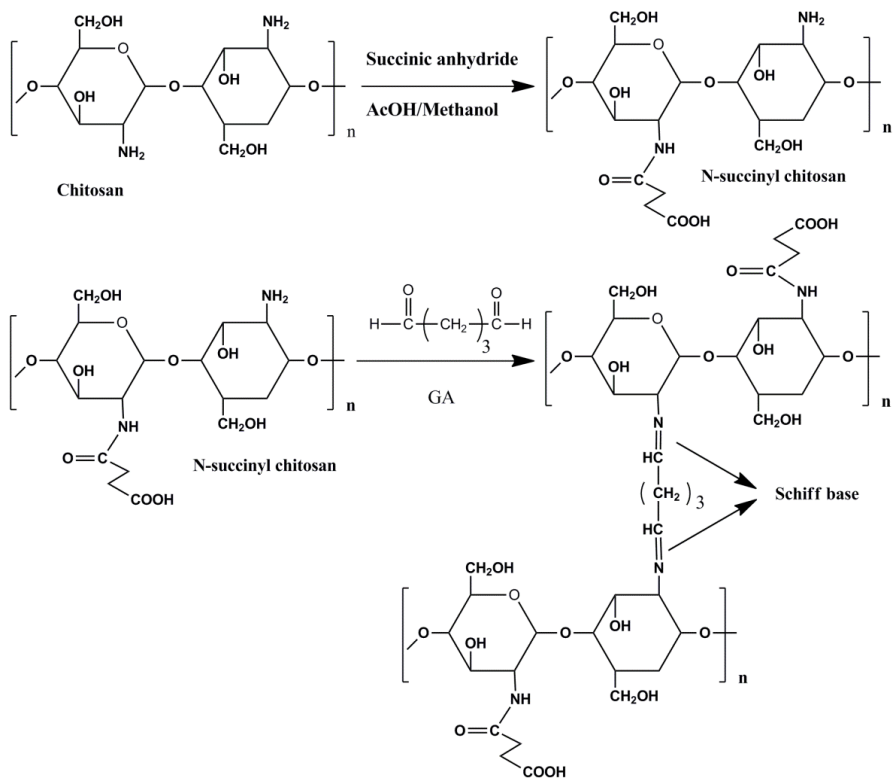

Figure 1. The proposed reaction mechanism of $\mathrm{N}$-succinyl chitosan and its hydrogel formation.

\subsection{Characterization}

\subsubsection{Fourier transform infrared (FTIR) Analysis}

Figure 2 represents the FTIR spectra of chitosan, NSC, and NSC hydrogel. In Figure 2 (a) the peaks $3360 \mathrm{~cm}^{-1}, 2880 \mathrm{~cm}^{-1}, 1585 \mathrm{~cm}^{-1}, 1148 \mathrm{~cm}^{-1}, 1064 \mathrm{~cm}^{-1}$, and $1029 \mathrm{~cm}^{-1}$ represent the characteristic peaks of O-H and N-H stretching vibration, axial stretching vibration of $\mathrm{C}-\mathrm{H}$, protonated amino group, saccharide unit of chitosan, $\mathrm{C}-\mathrm{N}$ and $\mathrm{C}-\mathrm{O}$ stretching vibration, respectively. The spectrum of NSC is shown in Figure 2 (b). From this spectrum, it can be seen the stretching vibration of $\mathrm{O}-\mathrm{H}$ and $\mathrm{N}-\mathrm{H}$ at $3318 \mathrm{~cm}^{-1}$ and axial stretching vibration of $\mathrm{C}-\mathrm{H}$ at $2950 \mathrm{~cm}^{-1}$. The peak at $1545 \mathrm{~cm}^{-1}$ shows the presence of secondary amide. Moreover, the new peak appeared at $1420 \mathrm{~cm}^{-1}$ is due to presence of COO- ions. Furthermore, the peaks at $1169 \mathrm{~cm}^{-1}, 1064 \mathrm{~cm}^{-1}$, and $1029 \mathrm{~cm}^{-1}$ are due to saccharide unit, $\mathrm{C}-\mathrm{N}$ and $\mathrm{C}-\mathrm{O}$ stretching vibration, respectively. N-succinylation provoked structural changes which can be clearly seen by comparing the spectra of chitosan and NSC. The significant evidence is the occurrence of new peak at $1401 \mathrm{~cm}^{-1}$ due to asymmetric stretching vibration of $\mathrm{COO}^{-}$ions. These results are in good agreement with already reported [15]. In Figure 2 (c), FTIR spectrum of NSC gel exhibits almost all the peaks of NSC with a peak at $1643 \mathrm{~cm}^{-1}$ attributes $\mathrm{C}=\mathrm{N}$ stretching vibration owing to the formation of Schiff base between amino groups of NSC and aldehydic groups of glutaraldehyde.

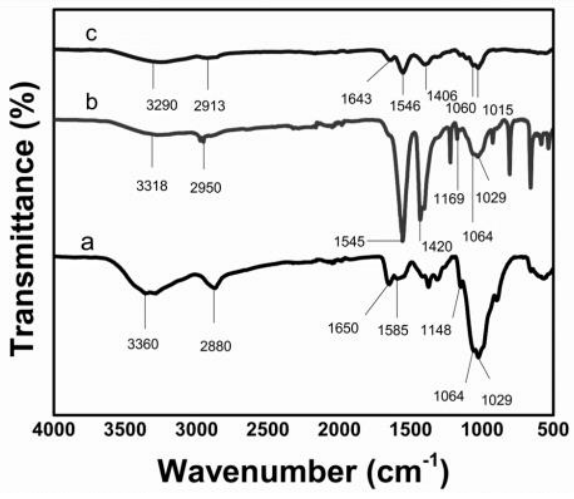

Figure 2. FTIR spectra of a) chitosan, b) N-succinyl chitosan, and c) Nsuccinyl chitosan hydrogel. 


\subsection{2. ${ }^{1} \mathrm{H}$ and ${ }^{13} \mathrm{C}$ NMR spectra of NSC}

The ${ }^{1} \mathrm{H}$ and ${ }^{13} \mathrm{C}$ NMR spectra of NSC are shown in Fig. 3. The peaks at 1.97, 2.37 , and $2.46 \mathrm{ppm}$ correspond to $-\mathrm{NH}-(\mathrm{CO})-\mathrm{CH}_{3},-\mathrm{NH}-(\mathrm{CO})-\mathrm{CH}_{2}-$ and $-\mathrm{CH}_{2}-$ $\mathrm{COO}$, respectively. The peak at $2.60 \mathrm{ppm}$ corresponds to $\mathrm{H}-2$ of glucosamine. The peaks from 3.45 to $3.77 \mathrm{ppm}$ correspond to chitosan backbone hydrogen. $\mathrm{H}-$ 1 of glucosamine unit is located at $4.4 \mathrm{ppm}$. The ${ }^{1} \mathrm{H}$ NMR confirms that the site for formation of NSC is at $-\mathrm{NH}_{2} \cdot{ }^{13} \mathrm{C}$ NMR spectrum of NSC shows that the peak at $22.12 \mathrm{ppm}$ represents $-\mathrm{NH}-(\mathrm{CO})-\mathrm{CH}_{3}$ while the large peaks at 32.41 and 32.69 ppm correspond to $-\mathrm{CH}_{2}-\mathrm{CH}_{2}$. The small peaks at 54.83, 60.05, 71.97, 74.59, 76.14, and 101.16 ppm confirm C-2, C-6, C-3, C-4, C-5 and C-1 of chitosan main chain respectively. The peak at 174 and $177.34 \mathrm{ppm}$ shows the presence of $-\mathrm{NH}$ (CO)- $\mathrm{CH}_{2}-\mathrm{CH}_{2}$ and $-\mathrm{COONa}$, respectively. These NMR spectral characteristics are in agreement with the literature [16, 17].

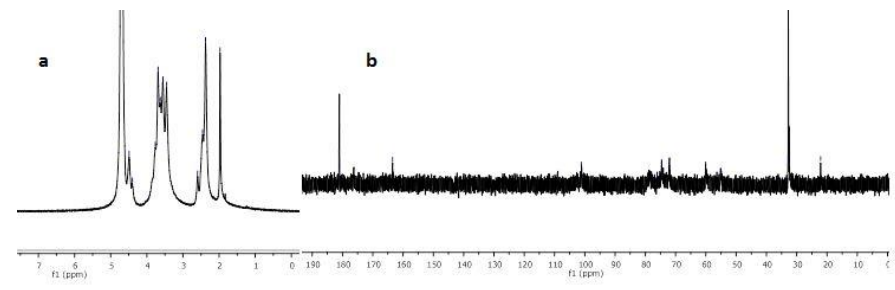

Figure 3. ${ }^{1} \mathrm{H}$ and ${ }^{13} \mathrm{C}$ NMR spectra of NSC

\subsubsection{X-ray diffraction (XRD) Analysis}

XRD patterns of chitosan, NSC, and NSC hydrogel is illustrated in Figure 4 (a, b \&c). In Figure 4 (a), there are reflection fall in chitosan pattern, one at $10^{\circ}$ and other at $20^{\circ}$. The weak reflection fall at $10^{\circ}$ assigns to crystal form I and strong reflection at $20^{\circ}$ shows the crystalline form II. These phases correspond to development of crystallinity in chitosan due to the hydrogen bond formation between amino and hydroxyl group and regularity in the chitosan structure. XRD pattern reveals that chitosan is partially crystalline polysaccharide due to regular chains [18]. The XRD pattern of NSC is shown in Figure 4 (b). In this pattern, it can be easily observed that reflection fall at $10^{\circ}$ disappeared and the intensity of peak at $20^{\circ}$ also decreased, indicating that chitosan structure was influenced during succinylation. During succinylation, inter-molecular hydrogen bonding breaks between amino and hydroxyl groups on chitosan chain and $\mathrm{COOH}$ group of succinyl exist as $\mathrm{COO}^{-}$ions. This is also verified from existing literature [19]. Furthermore, succinyl group is bulky as compared to $\mathrm{H}$ and it disturbs the regular crystal order and also increases the water holding capacity of NSC. With the increase in water holding capacity, amorphous nature also increases which results in decrease in crystallinity. The XRD pattern of NSC hydrogel is shown in Figure 3 (c). This pattern reveals further decrease in intensity at $20^{\circ}$ due to Schiff base formation between the crosslinking agent and NSC hence lead to destruction of inter and intra molecular hydrogen bonding and regularity in chitosan structure [20].

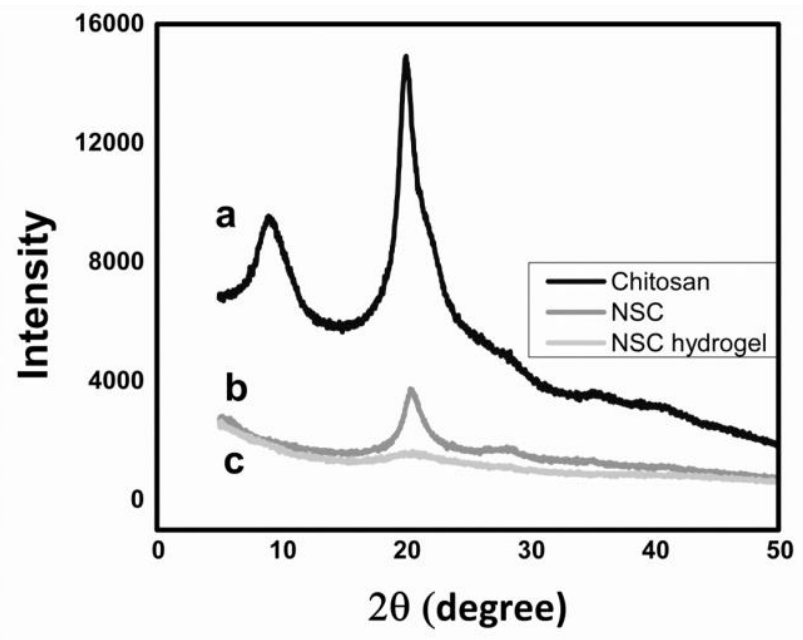

Figure 4. XRD spectra of chitosan, b) N-succinyl chitosan, and c) N-succinyl chitosan hydrogel

\subsubsection{Differential scanning calorimetry (DSC)}

Thermal behavior of chitosan, NSC, and NSC hydrogel was observed using DSC and results are summarized in Figure 5. Two curves were appeared in DSC analysis of chitosan; one is endothermic and the other is exothermic curve. The endothermic curve at a temperature of $152{ }^{\circ} \mathrm{C}$ represents the removal of bound water and transition of chitosan from solid crystalline state to soft rubbery state. This temperature is known as glass transition temperature $\left(\mathrm{T}_{\mathrm{g}}\right)$ of chitosan. The second exothermic curve at $300{ }^{\circ} \mathrm{C}$ corresponds to decomposition of chitosan. Furthermore, DSC thermogram of NSC also shows two curves. First endothermic curve at $144{ }^{\circ} \mathrm{C}$ represents the removal of bound water and transition from solid to soft rubbery state. However, exothermic curve at $268{ }^{\circ} \mathrm{C}$ is owing to thermal decomposition of NSC. The difference between endothermic curves of chitosan and NSC may be due to difference in molecular weight because chitosan modification occurred under alkaline condition and there are also chances of depolymerization in this condition [21]. The deep endothermic curve of NSC also shows the greater water holding ability due to more hydrophilic nature [22]. The broadness of endothermic curve also shows the greater amorphous nature of NSC which is already described in XRD results [23]. The chemical modification of chitosan also resulted in an increase in the content of sorbed water due to decrease in ordered structure [21]. Moreover, DSC thermogram of NSC hydrogel shows three endothermic curves, first one at $55^{\circ} \mathrm{C}$ represents removal of moisture contents and second endothermic curve with a peak temperature of $147{ }^{\circ} \mathrm{C}$ expressed the phase change from solid to rubbery state. Furthermore, third endotherm at a temperature of $244{ }^{\circ} \mathrm{C}$ shows the breakdown of crosslinking between NSC molecules. The crosslinked NSC did not show decomposition peak which is characteristic peak of chitosan polysaccharide [23].

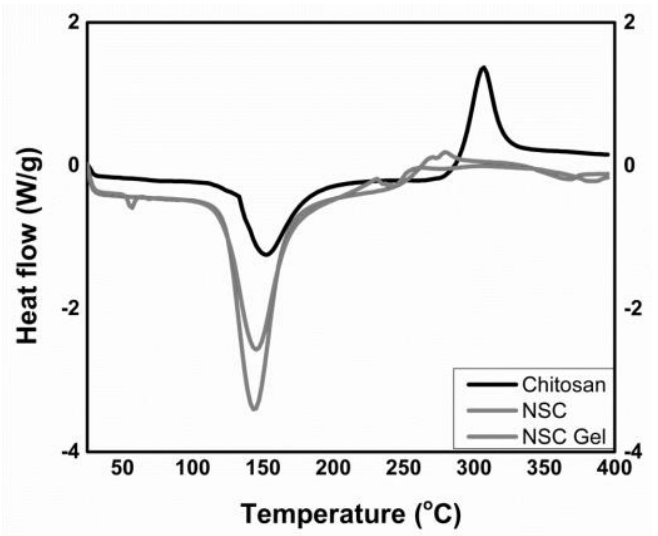

Figure 5. DSC thermograms of chitosan, $\mathrm{N}$-succinyl chitosan and $\mathrm{N}$-succinyl chitosan hidrogel.

\section{Field emission scanning electron microscopy (FESEM) analysis}

Field emission scanning electron microscope is very useful to observe the three-dimensional network structure of hydrogel. In this study, morphology of freeze-dried synthesized hydrogel was investigated to show porous network structure. The surface images of hydrogel are shown in Figure 6. From the images, it can be seen that hydrogel retain its porous network. It can imbibe water in large amount which was also confirmed from swelling ratio results. The hydrogels showed continuous and porous network structure by freeze-drying step. These images resembling other natural polymer hydrogels [24].
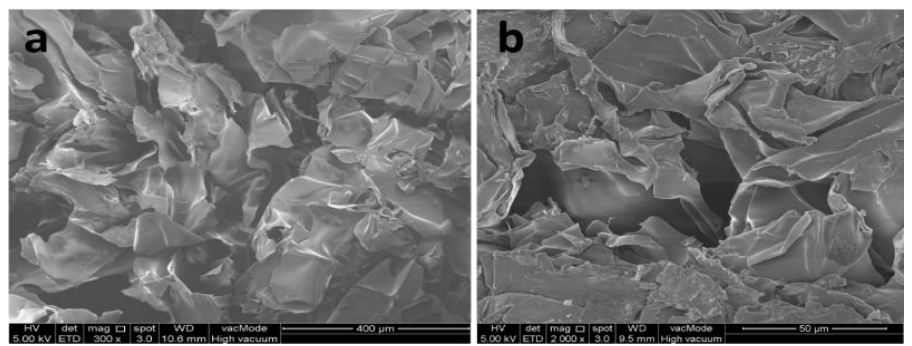

Figure 6. Micrographs of $\mathrm{N}$-succinyl chitosan hydrogel at low and high magnification 


\subsection{Rheology study}

Oscillatory linear frequency sweep test was conducted to evaluate the dynamic gel properties, namely, the stability of three-dimensional crosslinked network of hydrogels at constant strain. The hydrogels were subjected to frequency from 1 $\mathrm{Hz}$ to $50 \mathrm{~Hz}$. The frequency sweep profile of elastic modulus (G') and viscous modulus (G") is shown in Figure 7. The results revealed the dominant elastic behavior of the material over viscous nature which means the gel has formed after successful crosslinking of NSC chains. The rheogram shows the frequency dependence nature of the hydrogels which shows high mechanical strength at low frequency and it decreased with the corresponding increase in the frequency. The decrease in elastic behavior with the increase in frequency indicates the breakage of gel structure by mechanical shear. Their rheological behavior is typical of a 'weak gel', which is the frequency dependence [25]. The weak rheological behavior is also owing to natural polymer backbone. However, the elastic behavior of the hydrogel is dominant over viscous behavior throughout the frequency sweep. This feature is a characteristic of gel formation.

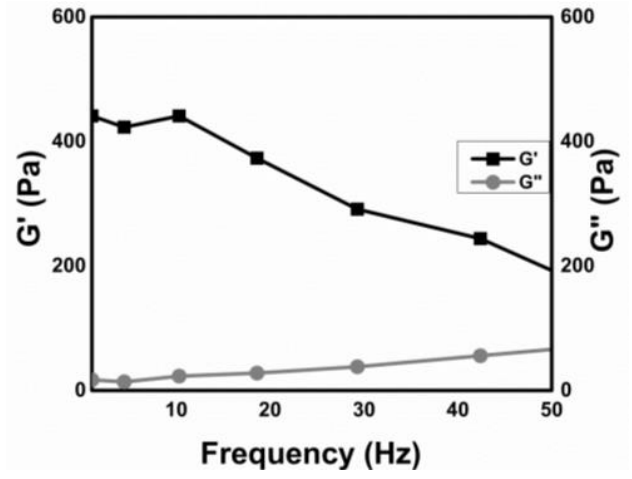

Figure 7. Elastic and viscous behavior of $\mathrm{N}$-succinyl chitosan hydrogel

\subsection{Swelling ratio study}

The synthesized hydrogel was observed for swelling in simulated gastric fluid (SGF, pH 1.2) and simulated intestinal fluid (SIF, pH 7.4) and results are summarized in Figure 8. The swelling of hydrogel primarily depends on the osmotic pressure difference between inside the gel and the surroundings caused by the redistribution of mobile ions. In this study, $\mathrm{pH}$ sensitive swelling ratio was observed and results indicated that hydrogel showed less swelling ability at $\mathrm{pH}$ 1.2 while high swelling ability at $\mathrm{pH}$ 7.4. This $\mathrm{pH}$ sensitive swelling behavior was due to the hydrophilic nature of NSC polymer and the presence of amino and carboxyl groups which form oppositely charged network structures. This charged structure might change the charge state of ionic groups with change in $\mathrm{pH}$. Furthermore, swelling at $\mathrm{pH} 1.2$ is due to presence of amino groups and swelling at $\mathrm{pH} 7.4$ is due to the existence of carboxyl groups and hydrophilic nature of NSC [26]. The low swelling ability at pH 1.2 is due to repulsion of protonated amino groups which are dominant at this condition. The number of amino group presence was reduced after chitosan modification and ultimately lower the swelling ratio. Moreover, the high swelling ability at $\mathrm{pH} 7.4$ is owing to the repulsion of carboxylate ions and hydrophilic polysaccharide backbone of NSC. The carboxylate ions are high in number and promote greater repulsion which resulted in high swelling ratio [27].

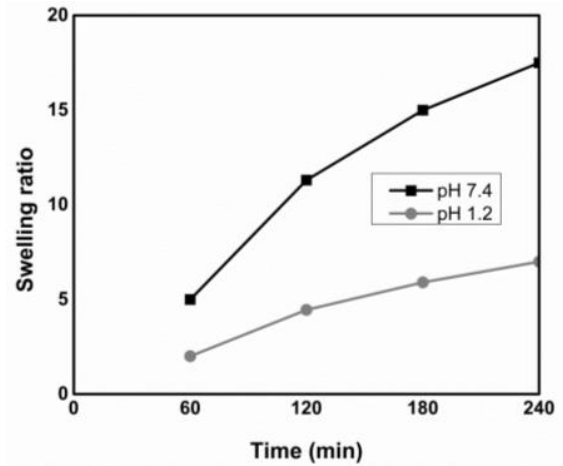

Figure 8. Swelling ratio of NSC hydrogel in buffer solutions of $\mathrm{pH} 7.4$ and $\mathrm{pH}$ 1.2 .

\section{CONCLUSION}

In conclusion, preparation of $\mathrm{N}$-succinyl chitosan was achieved successfully from $\mathrm{N}$-succinylation of chitosan and its hydrogel was synthesized through Schiff base crosslinking. The occurrence of NSC and Schiff base formation was confirmed using FTIR, XRD, and DSC. These results confirmed the successful synthesis of NSC and its hydrogel. Furthermore, the morphology study evidenced the highly porous structures of the hydrogel which can absorbs and retains large quantity of water due to its porosity property. Later on, the swelling properties also verified the porous network of the hydrogel. The hydrogel showed low swelling ratio at $\mathrm{pH} 1.2$ while high swelling ratio at $\mathrm{pH}$ 7.4. The change in the swelling ratio with the change in $\mathrm{pH}$ shows the $\mathrm{pH}$ sensitivity of NSC hydrogel. Moreover, the mechanical properties show that hydrogel behave as elastic material. Due to this property, it can be used in the biomedical applications, which is another significant evidence of its use in the biomedical field.

\section{ACKNOWLEGEMENT}

This work is financially supported by Fundamental Research Grant Scheme (FRGS) from Ministry of Education, Malaysia (FP062-2018A).

\section{CONFLICT OF INTEREST STATEMENT}

The authors have no conflict of interest statement.

\section{REFERENCES}

1. A.S. Hoffman, Adv. Drug. Deliver. 64, 18(2012).

2. D. Campoccia, et al., Biomaterials. 19(23), 2101(1998).

3. O. Wichterle and D. Lim, Nature. 185, 117(1960).

4. T.R. Hoare and D.S. Kohane, Polymer. 49(8), 1993(2008).

5. N. Peppas and A. Mikos, Hydrogels in medicine and pharmacy. 1, 1(1986).

6. N. Peppas, P. Bures, W. Leobandung, and H. Ichikawa, Eur. J. Pharm. Biopharm. 50(1), 27(2000).

7. C. Pillai, W. Paul, and C.P. Sharma, Prog. Polym. Sci. 34(7), 641(2009).

8. N. Bhattarai, J. Gunn, and M. Zhang, Adv. Drug. Deliver. 62(1), 83(2010).

9. X. Yang, Q. Liu, X. Chen, F. Yu, and Z. Zhu, Carbohyd. Polym. 73(3), 401(2008).

10. M. Izume, Chitin Chitosan Res. 4, 12(1998).

11. Y. Kato, H. Onishi, and Y. Machida, Biomaterials. 21(15), 1579(2000).

12. A. Golyshev, Y.E. Moskalenko, and Y.A. Skorik, Russ. Chem B+. 64(5), 1168(2015)

13. S. Bashir, Y.Y. Teo, S. Ramesh, K. Ramesh, and A.A. Khan, Rev. Chem. Eng. 31(6), 563(2015)

14. X. Liu, et al., J. Mater. Chem. B. 1(35), 4484(2013).

15. S. Bashir, Y.Y. Teo, S. Ramesh, and K. Ramesh, Polymer. 92, 36(2016).

16. I. Leceta, P. Guerrero, I. Ibarburu, M. Dueñas, and K. De la Caba, J. Food Sci. 116(4), 889(2013).

17. P. Mukhopadhyay, et al., Carbohyd. polym. 112, 627(2014).

18. G. Lu, et al., Eur. Polym. J. 43(9), 3807(2007).

19. C.d.T. Neto, et al., Carbohyd. Polym. 62(2), 97(2005).

20. F. Tang, et al., Int. J. Biol. Macromol. 93, 1995(2016).

21. M. Yar, et al., Mater Sci. Eng. B. 56, 154(2015).

22. H. Tan, C.R. Chu, K.A. Payne, and K.G. Marra, Biomaterials. 30(13), 2499(2009).

23. Y.-F. Tang, Y.-M. Du, X.-W. Hu, X.-W. Shi, and J.F. Kennedy, Carbohyd. polym. 67(4), 491(2007)

24. P. Li, J. Zhang, and A. Wang, Macromol. Mater. Eng. 292(8), 962(2007).

25. S.S. Vaghani, M.M. Patel, and C. Satish, Carbohyd. Res. 347(1), 76(2012).

26. Li, P., J. Zhang, and A. Wang, A Novel N-Succinylchitosan-graftPolyacrylamide/Attapulgite Composite Hydrogel Prepared through Inverse Suspension Polymerization. Macromol. Mater. Eng., 2007. 292(8): p. $962-$ 969.

27. Vaghani, S.S., M.M. Patel, and C. Satish, Synthesis and characterization of pH-sensitive hydrogel composed of carboxymethyl chitosan for colon targeted delivery of ornidazole. Carbohydrate research, 2012. 347(1): p. 7682. 